\title{
SYMMETRY IN MEASURE ALGEBRAS ${ }^{1}$
}

\author{
BY ARTHUR B. SIMON
}

Communicated by Walter Rudin, July 4, 1960

It is well known that the measure algebra of a locally compact group $G$ is not symmetric, i.e. the set of Gelfand transforms is not closed under complex conjugation. However, if these transforms are restricted to the character group $\Gamma$, they are symmetric. In his paper [1] Rudin asks: Is there a set larger than the closure of $\Gamma$ on which the transforms are symmetric?

If $G$ is the real line the answer is yes.

Let $G$ be the real line; we consider the algebra $M(G)$ of all regular Borel measures with convolution as multiplication. The maximal ideal space $\mathfrak{M}$ of $M(G)$ is compact and $\Gamma$ (also the real line) is an open subset of $\mathfrak{M}$. Let $S$ be the largest subset of $\mathfrak{M}$ on which the Gelfand transforms are closed under conjugation.

Let $Q$ be an independent, compact, perfect set (of Lebesgue measure 0 ) which supports a positive measure $\sigma$ whose Fourier-Stieltjes transform vanishes at infinity (see [2]). Without loss of generality we may suppose that the

$$
\sup \{|\hat{\sigma}(\gamma)|: \gamma \in \Gamma\}=\sup \left\{\left|\int_{-\infty}^{\infty} e^{i y x} d \sigma(x)\right|: y \in G\right\}=1 .
$$

Now let $U=\{h \in \mathfrak{M}:|\hat{\sigma}(h)|<1 / 4\}$. Since $\sigma$ vanishes at infinity the set $A=\Gamma-U$ is compact.

Pick an absolutely continuous measure $\lambda$ so that $\lambda \equiv 1$ on $A$.

We are now in position to define a member of $S$ which is not in the closure of $\Gamma$. We define a function to be identically -1 on all of $Q$ but one point $x$, and there its value is +1 . Since $Q$ is independent we can extend this function to a homomorphism $\chi_{\sigma}$ on $G$ to the circle group; since $Q$ is perfect $\chi_{\sigma}$ is not continuous but, clearly, $\chi_{\sigma}$ is $\sigma$-measurable. Now let $H=\left\{\mu \in M(G): \chi_{\sigma}\right.$ is $\mu$-measurable $\}$ and let $I=\{\phi \in M(G): \phi \perp \mu$ for every $\mu \in H\}$. Sreider [3] has shown that $H$ is an algebra, $I$ is an ideal and $M(G)=H+I$ (direct sum). Now pick $\chi_{0} \in A$ such that $\left|\hat{\sigma}\left(\chi_{0}\right)\right|>3 / 4$; and define $h_{0}(\mu)=\hat{\mu}\left(h_{0}\right)=\int \chi_{0}(x) d \mu_{H}(x)$, where $\mu_{H}$ is the projection of $\mu$ on $H$. It can be shown that $h_{0} \in S$ (since $H$ is self-adjoint) and that $\lambda \in I$ (since $\chi_{\sigma}$ is not continuous); thus if we let $W$ be the neighborhood of $h_{0}$ determined by $\sigma, \lambda$, and $1 / 4$, then $W \cap \Gamma=\phi$ and the result is proved.

1 This research was supported by the Air Force Office of Scientific Research Contract No. 49(638)383. 
REMARK. Obviously the more general theorem is true: Let $G$ be a locally compact abelian group. If there is a singular measure $\mu$ on $G$ whose (Gelfand) transform vanishes on the boundary of the character group $\Gamma$ and there exists a noncontinuous character on $G$ which is $\mu$ measurable, then $S \neq \bar{\Gamma}$.

\section{REFERENCES}

1. Walter Rudin, Measure algebras on abelian groups, Bull. Amer. Math. Soc. vol. 65 (1959) pp. 227-247.

2. - Fourier-Stieltjes transforms of measures on independent sets, Bull. Amer. Math. Soc. vol. 66 (1960) pp. 199-202.

3. Yu. A. Šreider, The structure of maximal ideals in rings of measures with convolution, Amer. Math. Soc. Translations no. 81, Providence, 1953.

NORTHWESTERN UNIVERSITY 